\title{
ESQUEMA PARA UN ANÁLISIS DEL PROCESO DE EXPANSIÓN DE LOS MOVIMIENTOS RELIGIOSOS PROTESTANTES EN EL PERÚ: ESTUDIO DE CASO EN LIMA
}

\author{
Israel Mandujano Caviedes \\ School of Mission and Theology, Stavanger, Noruega \\ israel_no@yahoo.com
}

\section{RESUMEN}

El presente análisis es un esfuerzo para conocer las formas y condicionamientos que han llevado a la expansión de los movimientos protestantes en el Perú, usando la referencia principal de la Alianza Cristiana y Misionera en el Perú, aunque no la única. Este análisis a la vez tiene el interés de proveer simultáneamente al lector-investigador de una metodología básica para profundizar en el estudio de estos movimientos, ya sea dentro del protestantismo o en otra tradición. Esta metodología básica cubre cuatro áreas principales que proveen un marco de observación e interpretación a los datos relacionados al tema de la expansión de estas iglesias. Las cuatro perspectivas fundamentales en este estudio por lo tanto son: la historia, la cultura, la ética y la teología. Estas cuatro subdivisiones de estudio pueden servir como instrumentos de análisis por temas, ya sea de manera independiente o interrelacionada.

\section{PALABRAS CLAVE}

Historia / Cosmovisión / Cultura / Etos / Teología.

\section{ABSTRACT}

This analysis aims at knowing forms and conditionings that have led to an expansion of protestant churches in Peru using the case of the Christian and Missionary Alliance, even though it is not the only one. This analysis carries also the interest of providing the student with a basic methodological approach to study these church movements and others in a deeper way. This basic methodology aims at covering four main areas, which provide a frame of observation and interpretation of the data related to the expansion of these churches. The four main approaches for this study are: History, Culture, Ethos and Theology. These four subdivisions of study can be used as tools to analyse these themes independently or interrelated.

\section{KEY WORDS}

History / Worldview / Culture /Ethos / Theology. 


\section{INTRODUCCIÓN}

Los movimientos protestantes en Latino América han experimentado un importante crecimiento en sus membresías y de manera especial desde los años 1970s según el PEW Research Center("Religion in Latin America : Widespread change in a historically Catholic region," p. 16). En el Perú estos movimientos de fe representan una gran variedad de estilos y denominaciones que conforman el ya notorio abanico de las iglesias protestantes.

Este movimiento, que se puede catalogar religioso, o de fe, constituye también un movimiento social enmarcado dentro del contexto histórico y demográfico de la sociedad Peruana. En este artículo nos ocupamos principalmente del crecimiento de la Alianza Cristiana y Misionera - AC\&M - en Lima, usándolo como un caso referencial para la comprensión de este fenómeno social evangélico, sin embargo, también haremos referencia a otros grupos del mundo protestante en el Perú.

En el proceso analítico me serviré de 4 formas de acercamiento para este estudio. El primero es la aproximación histórica, la cual nos ayudará a determinar los principales eventos que dieron lugar al desarrollo de la AC\&M. La interpretación de los eventos, es decir la lectura y la categorización de los acontecimientos internos y externos - el contexto en que se desarrolla esta iglesia, nos ayudarán a establecer las referencias y datos sobre los que se puede hacer un análisis histórico.

El Segundo paso del proceso analítico tiene que ver con la interpretación del carácter cultural que manifiestan estos movimientos, y que nos dan pautas sobre la familiaridad o alienación cultural de ellos con respecto a las formas culturales en el Perú. Es en este análisis donde no se puede obviar el proceso hermenéutico, es decir interpretativo, de las expresiones culturales de los Peruanos que se reflejan u olvidan durante las prácticas de fe en estas iglesias. Estas expresiones culturales a las que nos referimos usualmente se dan como símbolos; ya sea en forma de gestos, palabras, $\mathrm{u}$ omisiones que tienen un sentido comunicativo en el contexto Peruano, incluso Latino Americano. De allí que para entender los significados de los símbolos nos apoyaremos en la comprensión de ellos como elementos culturales que precisan de una interpretación, en este caso semiótica, la cual "se relaciona con todo aquello que se expresa como signo o señal [mi traducción]"(Eco, 1977, p. 7) y que lleva un significado esencial o relacional. 
El tercer análisis es de carácter ético. En este proceso ubicaremos no solo los pasos que se han desarrollado hacia una nueva ética, sino también los valores éticos fundamentales sobre los cuales descansa su etos o ethos. El etos es lo que define o señala el carácter, la personalidad moral de estos movimientos, no solo como formas o normas, sino como el fundamento de sus actitudes hacia la vida misma, aquello que los hace conocidos frente a los demás, o los distingue, y que al fin de cuentas resulta atractivo para unos y desdeñable para otros (Geertz, 1973, p. 303).

El cuarto paso del análisis es el estudio de los pensamientos de fe, su teología. Lo que ellos creen es en parte conocido para la mayoría de Peruanos, mas resulta necesario en nuestra búsqueda de la comprensión de estos movimientos una aproximación más cercana hacia los puntos más importantes de su fe; es decir el rol de Dios en su cosmovisión, la idea de Cristo y la importancia de lo que creen acerca de él, el concepto de la vida y del ser humano, incluyendo los rasgos de elementos imaginarios - metafísicos. La teología de estos movimientos, como los demás puntos que hemos mencionado, son áreas de estudio complejas y que podrían analizarse de forma independiente. La intención de este artículo es exponer y usar este método de investigación y análisis como referencia básica en nuestro objetivo de entender y estudiar estos movimientos que forman parte de nuestro universo social en el Perú.

\section{EL ANÁLISIS HISTÓRICO}

Si se usa una aproximación historiográfica en la identificación de eventos y datos en los anales históricos de la AC\&M del Perú, ${ }^{1}$ o de cualquier otra institución protestante, es necesario incluir un análisis de tipo reflexivo y analítico que interprete los procesos de la vida de esta institución que dichos datos registran (Van Seters, 1983, pp. 1, 2). En otras palabras, escribir historia no es escribir simplemente un registro de datos, que son la base ineludible, sino es el proceso de búsqueda de conocimiento y comprensión de las formas de vida que los datos registran, incluyendo el significado correlativo de los eventos. Como escribe JohnVan Seters: "Escribir Historia primariamente no consiste en reportar de modo preciso los eventos pasados. También considera la razón para traer de vuelta el

1 Muchos de los datos de la historia de la AC\&M fueron registradas por sus misioneros norteamericanos y se encuentran en los archivos de la oficina central en Colorado Springs. La oficina central de la AC\&M del Perú en Lima tiene ahora copias de la mayoría de estos registros que están originalmente en inglés. 
pasado y el significado que se da a dichos eventos[mi traducción]"(Van Seters, 1983, pp. 4, 5).

El otro elemento importante en el análisis histórico de los eventos dentro de un ambiente o sector social de una población, es la comparación con el contexto que ha rodeado dichos eventos. Es decir, ningún ente social constituido por un grupo de personas, en este caso de la AC\&M, se desarrolla aisladamente de su contexto social. Los eventos internos por lo general tienen una relación con los eventos externos, ya sea como influencia o reacción. De allí que tiene que haber una comparación de los datos históricos internos, desde la institución, y los datos externos de la sociedad en la cual se ha movido o existido(Van Seters, 1983, pp. 10, 11).

En el caso de la AC\&M se pueden ubicar 3 momentos de importancia histórica. El primero tiene que ver con su aparición en el Perú, con su trabajo en la zona rural del Perú, el segundo con la iniciación de su obra en Lima, y el tercero con el apogeo de su obra desde los años 1970s.

La primera etapa de la AC\&M empieza formalmente en 1925 con la llegada de un grupo de misioneros norteamericanos a la selva peruana, estableciéndose primeramente en Cahuapanas(Kowálchuk, 2000, p. 102), luego la misión seguiría rumbo a Huánuco. En este grupo cabe la mención especial de Raymond Clark, quien hizo algunos viajes de exploración anteriores(Clark, p. 287). Fue así que la AC\&M entró al Perú desde Brasil y por rio. ¿Por qué vinieron estos hombres? De acuerdo a algunos reportes de la época, el motor principal de su empresa se debió al anhelo misionero de compartir su fe y sus recursos(Curtis, 1998, pp. 66, 67). El ímpetu misionero norteamericano y europeo llevó a muchos a otras localidades y países no- occidentales en ese tiempo. Al principio, la AC\&M enfocó su trabajo en las zonas rurales del Perú, y por eso al llegar al distrito de Cahuapanas, en el departamento de Loreto, instalaron allí la primera base de su misión para esa región. Al llegar a Huánuco hicieron de esta ciudad andino-amazónica el Centro de operaciones para su obra en Perú en adelante, convirtiéndose así la AC\&M en una iglesia con una fuerte imagen provinciana en ese entonces.

Por otro lado, en esta etapa de su establecimiento en el Perú, la AC\&M tenía una filosofía de trabajo inter-denominacional(Van de Walle, 
2009$, p. 2) $)^{2}$ ya que su estructura norteamericana reflejaba su interés de trabajar no como una institución separada sino junto a otras organizaciones protestantes, siguiendo así su perfil de asociación misionera(Kessler, 2010, p. 206). Esta fue la razón por la que la AC\&M empezó su trabajo en el Perú en coordinación y junto con la Iglesia Evangélica Peruana(Kessler, 2010, pp. 184-185, 207). Sin embargo esta cooperación no duraría por mucho tiempo, ya que ambas instituciones se separaron el 25 de Noviembre de 1954 debido, según Juan B.A. Kessler, a razones partidistas de algunos misioneros extranjeros que causaron mucho dolor entre los miembros de dichas iglesias(Kessler, 2010, p. 213).Esta experiencia muestra el sobreénfasis denominación al que parece haber seguido a las organizaciones protestantes en el Perú. Esta característica probablemente se deba a un excesivo control y autoritarismo como parte dela estructura en algunas de estas organizaciones y también al carácter caudillista de algunos líderes religiosos, extranjeros y nacionales, considerados jefes institucionales o profetas modernos con una aurea de espiritualismo(Palomino, 2001, pp. 167, 225). En los últimos años esta diversidad y diferencias entre denominaciones protestantes han pasado a ser más notorias debido al crecimiento de numerosas congregaciones pentecostales y neopentecostales que en su gran mayoría creen recibir continuas revelaciones sobrenaturales y directas por parte de Dios, así como formulas particulares para obtener sanación y otros favores a través de poderes milagrosos de algunos individuos escogidos(Martin, 1990, p. 167).La consecuencia es que las iglesias o "hermanos" que no siguen estas prácticas son a veces consideradas de otra categoría. La AC\&M pertenece a una línea de iglesias evangélicas que enfatizan más bien la educación y formación a través de estudios bíblicos, clases y sermones con un mensaje Cristo-céntrico ( http:// www.awf.nu/about-us/introduction/ ). El otro énfasis de la AC\&M ha sido su vocación misionera y militante, que en el Perú y Latino América también ha sido una característica de la iglesia protestante en general y a través de su historia (Pérez Guadalupe, 2008, p. 226). La división de énfasis del mensaje evangélico y las tendencias caudillistas y de poder, parecen haber creado un antagonismo de formas, estilos e incluso de ideologías dentro del mundo evangélico nacional, formando así las corrientes evangélicas y una débil unidad.

2 Se entiende por denominaciones protestantes a las distintas organizaciones misioneras, que después de haber llegado al Perú formaron congregaciones o iglesias, que luego pasaron a reflejar el perfil y énfasis de la organización que las fundó. 
La segunda etapa de la obra de la Alianza en el Perú comienza a fines de los años 1950s. Desde entonces su obra se concentraría principalmente en urbes, siendo la capital Lima la que experimentaría un crecimiento sorprendente y especial. La obra en Lima se debió tanto a la necesidad de establecer una iglesia para miembros de esta iglesia que empezaron a inmigrar desde las provincias(LED, 1999, p. 19) como también al deseo de ver una iglesia nueva y limeña.

La inmigración mayor de gente desde las provincias del Perú a Lima se acentuó desde esta década - 1950s - hasta el presente(Matos Mar, 2005, p. 75). Esto hizo que la obra de la AC\&M en Lima empezara como una obra evangélica urbana y a la vez con una profunda presencia provinciana. En la capital, la AC\&M se estableció primero en uno de los barrios más acomodados de Lima, en el distrito de San Isidro. Luego comprarían una propiedad en Lince, que se conoce mas bien como un barrio de "clase-media," y en una de las avenidas más transitadas de Lima, la Av. Arequipa (C\&MA, 1958-, p. 52). Pronto se convirtió en una congregación de extranjeros, limeños y provincianos; de obreros y empresarios; con gente del centro y de la periferia de la ciudad. Esta diversidad parece haberle dado a la AC\&M un carácter cultural heterogéneo y urbanístico propio de la ciudad de Lima, que se convirtió en una ciudad basta e intercultural, espejo de diferentes culturas peruanas que se han fundido dándole un nuevo rostro a la Ciudad del los Reyes, es decir la de una sociedad profundamente variada y entusiasta (Arellano Cueva \& Abugattas, 2004, p. 73).

Sin embargo, no sería hasta mediados de los años 1970s cuando empieza el crecimiento notorio de miembros en la Alianza Cristiana y Misionera de Lima. Este fenómeno social marcaría el inicio de una tercera etapa, que sería una etapa de expansión, y que está durando hasta nuestros días. A principios de los años 1970s la AC\&M contaba con cerca de 117 miembros(Smith, 1994, p. 13). En 1974 la AC\&M con la representación principal en su iglesia del distrito de Lince pasó a contar con cerca de 400 miembros y una asistencia de 1000 personas(LED, 1999, p. 24). Esta es la tercera etapa, la del apogeo del crecimiento. Hoy la AC\&M consta con cerca de 40000 miembros y con cerca de 100 congregaciones en prácticamente cada distrito de Lima(Alianza Cristiana y Misionera).

La investigación hecha por el PewResearch Centre y publicada en el año 2014, muestra que la mayoría de los grupos protestantes comenzaron a crecer en membresía durante esta década de los años 1970 ("Religion 
in Latin America : Widespread change in a historically Catholic region," p. 26). Este dato sugiere que en el contexto social limeño hubo cambios o situaciones particulares en este periodo que condicionaron de manera general la apertura de los limeños hacia esta forma de fe. En el análisis del crecimiento, por lo tanto, es necesario considerar los condicionamientos sociales y externos como también los factores internos, y que resultaron atractivos como alternativas de fe en Lima y Latino América. Los condicionamientos internos fueron la fuerza de atracción que actuó como fuerza social centrifuga originada por el dinamismo del contexto exterior(Schreiter, 1985, pp. 7-8, 28-29). Otra forma de analizar ambas fuerzas de condicionamiento puede ser desde la perspectiva de las teorías de empuje/presión y atracción - 'push and pull' en inglés. Esta teoría de empuje y atracción ha servido para explicar, entre otros fenómenos sociales, la inmigración(Lee 1966). En este caso la fuerzas de empuje suelen ser situaciones de pobreza, hambre o inseguridad, que empujan a la gente a emigrar, mientras que la "buena" situación de los países a los cuales emigran, son las fuerzas de atracción. Por tal razón el crecimiento de las membresías de las iglesias evangélicas, de manera especial desde la década del 70, puede estudiarse desde esta perspectiva científica de los condicionamientos externos e internos que empujan y atraen para que la gente se adhiera a estas congregaciones desde entonces.

Un punto importante sobre los condicionamientos internos es la consideración de la variedad de formas, estilos y creencias dentro del mundo protestante que forman las distintas denominaciones. Mientras algunas congregaciones pueden atraer por lo sensacional de sus experiencias, otras atraen por apelar a la recepción y comprensión del mensaje de una forma cognitiva. Este fenómeno demuestra que no existe uniformidad entre las iglesias protestantes limeñas, y que sus estilos y énfasis pueden ser muy distintos. En el caso del crecimiento de las iglesias de la Alianza, su mensaje y formas parecen haber alcanzado a personas de diferentes trasfondos culturales, sociales y financieros, con un énfasis mayor en la confianza del conocimiento del mensaje presentado que en las expresiones sensacionales de sus experiencias. Históricamente podría alinearse la AC\&M con iglesias protestantes con énfasis en la evangelización, doctrina y su herencia como sociedad misionera.

\section{EL ANÁLISIS CULTURAL}

El análisis cultural nos lleva al terreno de las expresiones e ideas que conforman los rastros distintivos de un pueblo y que por lo general 
están en constante formación y cambios(Kraft, 1979, pp. 46-47). Los diferentes sentimientos y costumbres de los limeños, que se han ido formalizando a través de su historia, son hoy los elementos que componen su formación cultural, siendo su carácter híbridoo heterogéneo uno de ellos, y que han resultado en la multitud de tradiciones que se han juntado en su espacio social, especialmente después de las olas de inmigración provinciana a Lima desde los años 1950s. Otro elemento importante de la formación cultural limeña es su tradición religiosa que ha hecho de la sociedad limeña un pueblo inclinado a la creencia de la existencia de Dios sin mayores conjeturas. Como escribió don Manuel Marzal, que "en el Perú el ateísmo no es una experiencia común"(Marzal, 1996, p. 81). Esto indica la predisposición del limeño y el peruano a creer en Dios y ver la religión en general como parte natural de su existencia, o, como también lo señala Jeffrey Klaibber, "los Peruanos son profundamente religiosos"(Klaibber, 1996, p. 13).

En nuestro análisis cultural podemos señalar primero que el hibridismo cultural limeño se nota en áreas como el arte, la cocina, los matrimonios mixtos, o el lenguaje. En el área artística existen diferentes combinaciones musicales que son formas de fusiones entre los distintos géneros, como la chicha, la cumbia, la marinera, incluso el rock y el huayno. Dentro de la cocina, el abanico de platos peruanos es una combinación de orígenes diversos, como el lomo saltado, la papa a la huancaína, o el ceviche. La cocina peruana se ha convertido hoy una de las máximas expresiones de su fusión cultural (Adriá, 2011). La gran mayoría de familias son el resultado de encuentros de parejas con distinta procedencia regional(Bruce, 2008 (primera edición en 2007), p. 32)y por lo tanto cultural(Schmidt, 2014, pp. 29-31). Esta diversidad y fusión matrimonial se pueden observar entre otras formas, por la variedad de los apellidos de origen español y quechua. Como Gonzales y Mamani. El lenguaje común limeño es el Castellano, sin embargo se habla de muchas maneras y con muchos acentos diversos a causa de la procedencia provinciana, donde aparte del Castellano hay otros idiomas. Estas originalidades de la sociedad limeña hacen que su población sea culturalmente mixta. La pregunta es si las iglesias protestantes son reflejo de esta diversidad de culturas hibridas en la capital y en el Perú. Hasta los años 1970s la gran población de las iglesias protestantes eran identificadas con la zona periférica, es decir con gente de origen y contexto muy modesto y provinciano. Sin embargo en la últimas décadas las iglesias protestantes en general son reflejo de la diversidad cultural y social de nuestras urbes, incluyendo zona altas y gente 
de trasfondos diferentes; incluso hay representantes evangélicos en el área política peruana("Pastor Humberto Lay vive de lo que le paga su iglesia,"). La Alianza tiene hoy iglesias prácticamente en cada distrito de lima, en zonas altas y zonas periféricas (LED, 1999, p. 88).Esto muestra que la AC\&M tiene un perfil que se adapta a las distintas zonas donde encontramos personas de distintos trasfondos culturales o sociales. No es una iglesia adaptada solo para gente con trasfondo económico modesto o alto, sino indistintamente. Culturalmente la AC\&M tiene un perfil incluyente que permite a las personas formar parte de ella sin exigencias de asimilación social. Esto se puede distinguir por el carácter nacional de la denominación y por la variedad de su música, que es internacional y también nacional. Sin embargo es preciso señalar que, el espacio para la música de carácter provinciano y andino sigue siendo limitado. La mayoría de las canciones que se usan son traducciones de canciones de origen anglosajón.

La AC\&M en el Perú hoy día es una organización eclesiástica independiente, es decir, que ya no depende ni económica ni culturalmente de la misión original norteamericana. En Lima hay pastores con trasfondos culturales y nacionales diversos y el racismo no es una práctica que la iglesia acepte. En esta denominación es posible encontrar gente con ingresos económicos relacionados a trabajos profesionales, como aquellos que tienen un ingreso modesto por su situación precaria que todavía es parte de la realidad peruana. Esta denominación no usa esta variable como razón para dividir sus iglesias. Tampoco es requisito ser de una zona geográfica particular o tener un lenguaje castizo o costumbres castizas para ser miembro de estas iglesias en Lima. Esto demuestra el alto nivel de representación de cultura hibrida limeña y de inclusión en estas congregaciones.

El otro rasgo cultural limeño y peruano es la herencia religiosa, trasmitida especialmente a través de la tradición católica. La relación entre un sistema cultural y la religión en sociedades religiosas y con cosmovisiones donde lo trascendental es aceptado como parte natural de su estructura, es estrecha. Esta visión cultural religiosa que se usa en la lectura analítica de una sociedad ha sido desarrollada particularmente por Emilie Durkheim y Max Webber. Ellos señalan que el proceso histórico acentúa las costumbres, especialmente cuando son prácticas de toda una sociedad en forma colectiva (Durkheim, 1968 - first edition in 1912, pp. 416-419), lo que formaliza la relación entre una cultura y su expresión religiosa a través de símbolos y significados consensuados (Weber, 1966 - first edition in 1920 , p. 6). Esto ocurre en la estructura correlativa que surgen entre 
elementos religiosos y sociales como las procesiones, peregrinaciones y las fechas que se convierten en especies de días sagrados, social y religiosamente a la vez.

Esta familiarización con elementos trascendentes parece haber predispuesto a la sociedad limeña a una nueva forma de Cristianismo, el protestante. Figuras del Cristianismo como la familia sagrada, Jesús, los profetas, incluso algunos dogmas no son relatos desconocidos para los peruanos. De allí que se puede sugerir que el cambio a esta nueva forma de Cristianismo ha dependido en gran parte al pre-conocimiento con el mensaje dado a través de la Iglesia Católica. Sin embargo es preciso señalar que el cambio de la iglesia católica por la iglesia protestante también puede deberse a la nueva forma de aproximación a la fe, es decir, como reacción a formas tradicionales. Por ejemplo, mientras que la fe cristiana expresada a través de la iglesia católica enfatiza la colectividad, la iglesia protestante enfatiza la individualidad, por eso es común escuchar en las iglesias evangélicas señalar a Cristo como "salvador personal." Este énfasis tiene relación con la Reforma Religiosa del siglo XVI en Europa, donde la experiencia personal de la fe se convirtió en un criterio básico del movimiento(Bosch, 2006 first edition in 1991, p. 242). Como conclusión, el catolicismo limeño y peruano cedió mucho de su mensaje al protestantismo novedoso, donde ambas fes tienen la plataforma común de la aceptación de lo trascendente como parte normal de su existencia. Semióticamente la devoción mantiene muchas de sus características de manera similar. Mientras que en la fe católica, se siguen ritos y peregrinajes, en las iglesias protestantes hay una militancia evangelizadora y formas de adoración, que en muchas iglesias evangélicas pentecostales y carismáticas toman expresiones de carácter numinoso (https://en.wikipedia.org/wiki/Numinous). En otros casos, se compara la necesidad de amuletos en la religiosidad católica con el uso de la Biblia como objeto concreto de protección entre los evangélicos, y también con el uso de términos 'sagrados' con atributos de poder.

\section{EL CARÁCTER ÉTICO}

Mientras el carácter ético-la moral-es el fundamento que componen los valores de una sociedad o persona, la moralidad podría entenderse como la practica en razón de esos valores. Ambas tienen una relación indivisible. Si la ética de una sociedad se basa en valores de justicia, solidaridad, amor y bondad, las obras de moralidad en dicha sociedad mostrarán esos valores. De no ser así habrá una incoherencia dicótoma que atomizará- desintegrará 
- la personalidad de tal sociedad o individuo, lo que también se conoce como corrupción o deterioro de la moral.

El Cristianismo en su esencia es no solamente un movimiento religioso sino un movimiento ético, que registra sus estándares morales basados en la moral de su fundador, Cristo. Estos estándares morales relacionados a las enseñanzas y vida del fundador del Cristianismo se encuentran registrados en los evangelios, siendo el principal documento didáctico de su moral el "sermón del monte." ${ }^{3}$ En una parte de ese sermón Cristo dijo a sus discípulos, "si vuestra justicia no fuere mayor que la delos escribas y fariseos, no entraréis en el reino de los cielos," haciendo alusión a la dicotomía entre la moral que enseñaban los fariseos, o líderes religiosos judíos de su tiempo, y sus formas de tratar a los demás. A la luz de esta forma integral de ver la vida y la fe por parte de Cristo, ¿cuál es el etos de estas instituciones protestantes o evangélicas que se presentan como seguidores suyos? Para responder de manera analítica, podemos limitarnos a tres valores morales básicos, 'la justicia o derecho,' 'el amor' y 'la bondad.'

El análisis solo nos permite probar la integridad de sus acciones en relación a sus etos - en plural y de forma limitada por la diversidad de expresiones dentro de los varios movimientos protestantes en Lima - pero sí se puede señalizar la coherencia entre los énfasis y las practicas.

En cuanto a la justicia, en muchas de las iglesias protestantes ha habido un cambio en cuanto a la valoración de la mujer como individuo y no solo como complemento matrimonial del hombre sin capacidades para gobernar o ejercer obras de liderazgo. De esta manera ser del género femenino ya no es razón para discriminar la mujer de cargos de liderazgo. En una sociedad como la peruana donde el androcentrismo - o machismo en el lenguaje popular - es la forma generalizada de relación de géneros, este cambio es un logro y un paso importante hacia la interacción de hombre y mujer en igualdad de derechos. La AC\&M por ejemplo ya tiene ministras registradas dentro de su organización, y otras instituciones tienen pastoras además de pastores. De este modo las iglesias evangélicas de Lima reflejan su carácter tradicional reaccionario, como fue el inicio de la Iglesia Metodista en el Perú (Gonzáles, 2008, p. 253),frente al status quo de una sociedad que estiman injustay que no aceptan. Lo otro es la diversidad

3 En el Evangelio según San Mateo, capítulos 5, 6 y 7.

4 Evangelio según San Mateo 5: 20. 
de trasfondos en la inclusión de sus miembros. Es difícil encontrar en Lima una institución protestante donde se discrimine a sus miembros por el trasfondo modesto o regional (LED, 1999, p. 64). ${ }^{5}$

En cuanto al amor al prójimo, de acuerdo al reporte del Pew Research Centre, las iglesias protestantes están envueltas en Latino América en acciones de solidaridad hacia los necesitados("Religion in Latin America : Widespread change in a historically Catholic region," p. 10),lo que muestra un deseo de seguir la ética de su maestro, Cristo, que dijo, "Como el Padre me ha amado, así también yo os he amado; permaneced en mi amor," ${ }^{\text {, } y}$ su identificación con el más débil en la sociedad. Este amor se nota especialmente en los modos como las iglesias protestantes se han convertido en refugios de acogida para mucha gente menos favorecidas de las urbes latinoamericanas("Religion in Latin America : Widespread change in a historically Catholic region," p. 37), lo cual Samuel Escobar también sostiene (Escobar, 2002, p. 27). Esta señal de acogida se nota en la gran mayoría de iglesias evangélicas entre las que también está la AC\&M, que además de tener iglesias en zonas altas de Lima, también tiene congregaciones en distritos de la periferia de la ciudad. Sin embargo, a la vez, es necesario señalar que algunas instituciones tienen problemas para salir de una comprensión extremadamente jerárquica de gobierno, lo que genera formas de represión. Hay casos conocidos donde líderes de algunas comunidades evangélicas han sido influenciados por la búsqueda de lujo o poder(Palomino, 2001, p. 28), exigiendo en algunos casos a los miembros aportes económicos que sobrepasan sus posibilidades y a cumplir sus obligaciones para con la iglesia ofreciendo sus propiedades (http://elcomercio.pe/lima/ciudad/secta-pare-sufrir-mueve-peru-us15-millones-diezmos-noticia-327637). Esto es una forma de opresión, sobre todo si se usa la religión como medio. Hoy en día en Lima el nivel financiero de muchos ha subido(Gestión, 2014), y esto se refleja en muchas nuevas congregaciones protestantes, sin embargo, esto es diferente a usar exigencias económicas usando amenazas en términos religiosos. Esta práctica se convierte en una forma de violentar la integridad y el derecho de una persona sobre sus ingresos y propiedades. Aun cuando estos casos son aislados, sin embargo, forman parte de una práctica con justificación ideológica en algunos sectores del mundo protestante.

5 Los pastores actuales de la AC\&M son de muchos trasfondos sociales y culturales.

6 En el evangelio según San Juan, 15:9. 
En cuanto a la bondad, en su sentido más fundamental, esta tiene que ver con la empatía que despierta el amor y que se expresa en actitudes y obras de bien en oposición a las obras de maldad. En esta área ética las iglesia evangélicas son por lo general sensibles a los extremos de maldad ciudadana mostrados en abusos físicos, violencias y explotaciones al prójimo. La búsqueda de lo bueno es un principio universal que va de la mano con el principio del derecho y el amor. Algunas de estas iglesias por ejemplo han desarrollado programas para gente en situación de pobreza("Religion in Latin America : Widespread change in a historically Catholic region," $\mathrm{p}$. 22). Durante la época del terrorismo, muchas de estas iglesias ubicadas en zonas de emergencia mantuvieron su integridad rechazando la invitación, y algunas veces la imposición, a unirse a grupos terroristas. Esto costó la vida a varios de ellos. Incluso la imposición de grupos terroristas a dar "cuotas revolucionarias" que se pedían y otras amenazascontra iglesias evangélicas no tuvieron éxito(López, 1998, pp. 129-137). Las iglesias evangélicas resistieron el chantaje que se quería ejercer sobre ellos. Recordadas son las marchas a favor de la paz en el Perú y las oraciones levantadas por el Perú. Asistieron con comisiones gestionar y trabajar por la paz social y vencer la tragedia del terrorismo en el Perú(López, 1998, pp. 137-138).

La AC\&M cuenta hoy día con instituciones educativas, programas de apoyo social y con una infraestructura que cubre prácticamente todos los distritos limeños y que le permite tener acceso a todos los niveles de nuestra sociedad limeña y peruana. Por otro lado, el celo evangelizador de sus miembros los hace militantes en su búsqueda de alcanzar al prójimo con las bondades y recursos de los que ellos disponen.

\section{LA TEOLOGÍA}

La orientación teológica es el sistema ideológico que sostiene la fe y expresiones religiosas que se pueden observar en las iglesias. Lo primero que se puede notar en estas iglesias evangélicas es el énfasis en los principios que ellos sostienen están basados en el mensaje bíblico. Sin embargo es importante señalar que las distintas tradiciones cristianas aparte de la evangélica, como la católica, también dicen ceñirse a estos principios derivados de la Biblia. Además, dentro del grupo de iglesias evangélicas, interiormente, existen posiciones teológicas muy variadas, incluso opuestas. Esto demuestra que no hay una línea clara y uniforme en todos los principios de las instituciones cristianas. A la vez, la mayoría de las iglesias evangélicas registran una actitud de respeto y búsqueda 
de comprensión del mensaje Biblio-céntrico y Cristo-céntrico, es decir principios basados en la historia de salvación registrada en la Biblia, con énfasis central en la vida misma de Jesucristo como el hijo de Dios, lo que se conoce como el Evangelio (Stott, 1971 - first edition in 1958 p. 12). Esto hace que la comprensión de ese mensaje del Evangelio, tiene como finalidad llevar al miembro a una comunión con Cristo de modo personal. Este énfasis del uso de la teología como instrumento para alimentar la fe personal, convierte la reflexión en una práctica dinámica no solo reservada a los líderes y teólogos oficiales sino también a los miembros comunes de estas iglesias. Aunque a la vez, en muchos casos, existe una repetición de principios o normas aprendidas de memoria sin mediar mucha reflexión.

La práctica teológica como un instrumento para toda la comunidad y no solo de los expertos, parece haber creado al menos dos líneas de teología, incluso en algunas iglesias evangélicas, y que son, la línea oficial y la línea popular. Para analizar las similitudes y diferencias entre ambas se puede echar mano de la obra del Dr. Manuel Marzal sobre Catolicismo Popular. Marzal hace una interesante diferenciación entre las dos teologías. La oficial es la que anuncia y representa las posturas ideológicas de la institución de manera universal, mientras la popular se forma en las bases populares, entre la gente local, con su propio entendimiento como marco de su fe. En este último caso la teología es profundamente contextual, lo que de acuerdo a Marzal ha llevado a los miembros a un proceso de síntesis de puntos de vistas o cosmovisiones de lo nativo y lo universal, de la religiosidad pre-hispana y el Catolicismo. Esta síntesis de cosmovisiones ha hecho que elementos de fe locales permanezcan, como las creencia de almas y espíritus caminando por las noches, supersticiones, las creencias en los curanderos, la auto flagelación. ¿Dónde se puede apreciar lo popular entre los evangélicos peruanos? La forma más notoria se da entre los movimientos neo-pentecostales con sus énfasis en las sanidades dramáticas, la gran admiración de sanadores relacionados a un nivel especial de espiritualidad según ellos. De allí que en la mayoría de estas iglesias, antes que un afán de conocer la doctrina o dogma de sus iglesias, más bien el énfasis se centraliza en las experiencias religiosas y de tipo 'numinoso.' Para muchos de ellos esas experiencias son la prueba de la presencia de Dios. Lo que el curandero es para el 'catolicismo popular,' el sanador lo es para el 'protestantismo popular'(Martin, 1990, p. 32), lo que también es sostenido por Asle Jøssang (Jøssang, 2010, p. 39). Lo que las peregrinaciones y procesiones con altos niveles de dramatismo significan para el catolicismo popular, en el protestantismo lo son los cultos de 
sanación en iglesias donde los "profetas" revelan el futuro y sanan por el poder que tienen - su estima es parecida a los santos locales de la Iglesia Católica en el Perú. En algunos contextos protestantes, si el sanador del culto no funciona, se probará con el curandero o chamán del pueblo, en una acción de perfecta síntesis religiosa.

En las iglesias de la AC\&M del Perú ha habido desde sus comienzos énfasis en el valor de la enseñanza y la comprensión cognitiva de ella como parte de su etos (LED, 1999, pp. 38-39), sin embargo su énfasis misionero y de obra evangelizadora ha hecho que esta iglesia en realidad no tenga documentos extensos ynormativos de teología, como en el caso de los luteranos que tuvieron a Martin Lutero, o los reformistas con Ulrico Zuinglio y Juan Calvino. Por esta razón en la AC\&M el elemento cohesivo ha sidosobretodo el ímpetu misionero y el énfasis al estudio bíblico. La debilidad de este sistema teológico radica en la extremada dependencia en el énfasis del pastor de turno en la iglesia local, cuyo mensaje se convierte en la referencia teológica, quien en la practica precisa mayormente señalar al énfasis Cristo-céntrico, expresado en su obra salvadora, sanadora, santificadora y su promesa de retornar y recoger a los que le esperan (http://www.cmalliance.org/about/beliefs/fourfold-gospel), en una forma de evangelio cuadrangular. Aparte de este énfasis no hay una teología oficial normativa como la que existe en otras tradiciones protestantes. En resumen, su teología es altamente practica y misionera, queusa la citación bíblica como una constante en la comunicación de su fe. Por lo tanto, la tradición teológica de la AC\&M en el Perú aunque sistemáticamente débil, sin embargo es enfática en su Cristología como fuente salvadora en la comprensión de la fe.

\section{CONCLUSIONES}

La primera conclusión tiene que ver con el carácter dinámico de la relación entre una sociedad y sus movimientos, en este caso los religiosos. Ninguno de los eventos que suceden de manera histórica entre los movimientos en estas categorías están divorciados de su contexto social y cultural, ya sea en forma de influencia o reacción.

La segunda conclusión tiene que ver con la forma como la mayoría de estos movimientos protestantes se han alineado con formas culturales natas de la idiosincrasia peruana, como lo es el carácter colectivo de su interés evangelístico y de la acogida que se nota en muchos de estas 
iglesias. De allí que la fe se entienda, aunque personal, como una manera de compartir con los demás.

La tercera conclusión tiene que ver con la relación, que en la mayoría de estas iglesias se busca, entre su fe y su manera de mantener valores. De allí que formas de inmoralidad no pertenecen al etos que históricamente les ha representado.

La última conclusión nos deja ver que en el Perú, en general, la mayoría de las iglesias protestantes han puesto el énfasis más en sus formas prácticas que en sus reflexiones teológicas. Probablemente esto ha llevado a un sinfín de variedades entre ellas, y que hoy hace difícil que se les defina en relación al énfasis de su fe.

\section{REFERENCIAS BIBLIOGRÁFICAS}

ADRIÁ, Ferrán

2011 Hay un fenómeno increible en el Perú. En: http://elcomercio.pe/gastronomia/1308200/noticia-ferran-adri-hay-fenomenno-increibleperu (Fecha de acceso: 23.10.2015).

ALIANZA CRISTIANA Y MISIONERA DEL PERÚ

2012 Estadística Nacional de la Alianza Cristiana y Misionera en el Perú - 2012. Lima, Perú: Consejo Directivo Nacional.

ARELLANO CUEVA, Rolando \&BURGOS ABUGATTAS, David 2004 Ciudad de los Reyes, de los Chávez, los Quispe. Lima: Empresa Periodística Nacional S.A.

BOSCH, David J.

2006 Transforming mission : paradigm shifts in theology of mission. Maryknoll, N.Y.: Orbis Books.

BRUCE, Jorge

2008 Nos habíamos choleado tanto : Psicoanálisis y racismo. Lima: Fondo Editorial/Universidad de San Martín de Porras.

C\&MA Lince

1958 Actas de la Iglesia. Lima. 
GONZALES, Justo L. \&CARDOZA, Carlos F.

2008 Historia general de las misiones. España: Editorial CLIE.

CLARK, Raymond B.

1924 Our advance to the forest Indians of Peru. USA: The Alliance Weekly, pp. 287-288, 295.

CURTIS, Carolyn

1998 A man for all nations. Camp Hill, Pennsylvania: Christian Publications, Inc.

DURKHEIM, Émile

1968 The elementary forms of religious life. London: George Allen \& Unwin LTD.

ECO, Umberto

1977 A theory of semiotics. London: Macmillan.

\section{EL COMERCIO}

2015 Secta Pare de Sufrir mueve en el Perú US\$1,5 millones por diezmos. En: http://elcomercio.pe/lima/ciudad/secta-pare-sufrir-mueve-peru-us15-millones-diezmos-noticia-327637 (Fecha de acceso: 24.10.2015).

\section{EL COMERCIO}

2010 Pastor Humberto Lay vive de lo que le paga su iglesia. En: http:// elcomercio.pe/politica/gobierno/pastor-humberto-lay-afirmavive-lo-que-le-paga-su-iglesia-noticia-483806 (Fecha de acceso: 24.10.2015).

ESCOBAR, Samuel

2002 Changing tides : Latin America and world misión today. Maryknoll, N.Y.: Orbis Books.

\section{GESTIÓN}

2014 FMI:Desarrollo económico del Perú comienza con los pequeños proyectos. En: http://gestion.pe/economia/fmi-parte-desarrolloperu-comienza-proyectos-pequenos-2115792 (Fecha de acceso: 23.10.2015). 
GEERTZ, Clifford

1973 The interpretation of cultures. New York: Basic Books.

GONZÁLES, Justo \& Carlos F. Cardoza

2008 Historia general de las misiones. España: Editorial CLIE.

JØSSANG, Asle

2010 Searching for a powerful Christ : An anthropology of religious conversion in Bolivia. (PhD), University of Oslo, Oslo.

KESSLER, Juan B.A.

2010 Historia de la evangelización en el Perú. Lima: Ediciones Puma.

KLAIBBER, Jeffrey L.

1996 La iglesia en el Perú. Lima: Fondo Editorial de la Pontificia Universidad Católica del Perú.

KOWÁLCHUK, Federico

2000 A Dios sea la gloria: Historia de la Alianza Cristiana y Misionera del Perú (1893-1999).

KRAFT, Charles H.

1979 Christianity in culture : a study in dynamic biblical theologizing in cross-cultural perspective. Maryknoll, New York: Orbis Books.

LED

1999 Libro memoria : Lima al encuentro con Dios [LED] XXV aniversario 1973-1998. Lima: Arte y Proyecto Gráfico.

LEE, Everett S.

1996 A theory of migration. Demography, vol. 3, no. 1, pp. 47-57.

LÓPEZ, Dario

1998 Los evangélicos y los derechos humanos. Lima: Ediciones Puma.

MARTIN, David

1990 Tongues of fire. Oxford: Basil Blackwell.

MARZAL, Manuel

1996 The indian face of God. New York: Orbis Books. 
MATOS MAR, José

2005 Desborde popular y crisis del estado : Veinte años después. Lima, Perú: Fondo editorial del congreso del Perú.

PALOMINO, Miguel

2001 The rise and face of neopentecostalism: A descriptive and comparative assestment of new independent charismatic/pentecostal churches in Peru. (PhD), University of Edinburgh, Edinburgh.

PÉREZ GUADALUPE, José Luis

2008 Y sin embargo se van... En Norberto Strotmann \& José Luis Pérez Guadalupe (Eds.), La iglesia después de 'Aparecida': Cifras y proyecciones (pp. 135-294). Lima: Talleres Gráficos de la Diócesis de Carabayllo.

\section{PEW}

2014 Religion in Latin America : Widespread change in a historically catholic región. En: http://www.pewforum.org/files/2014/11/Religion-in-Latin-America-11-12-PM-full-PDF.pdf (Fecha de acceso: 24.10.2015).

SCHREITER, Robert

1985 Constructing local theologies. Maryknoll, N.Y.: Orbis Books.

SCHMIDT, Jurge H.

2014 La comunicación intercultural: El desafio de la comunicación entre dos culturas. Norderstedt, Alemania: BoD Books on Demand.

SMITH, Alfredo C.

1994 Veinte años más tarde. Lima: Servicios Gráficos Smith-Marroquín.

STOTT, John

1971 Basic Christianity. Illinois: Inter-Varsity Press.

THE ALLIANCE

2015 Fourfold gospel. En: http://www.cmalliance.org/about/beliefs/fourfold-gospel (Fecha de acceso: 23.10.2015). 
THE ALLIANCE WORLD FELLOWSHIP

2015 About us. En: http://www.awf.nu/about-us/introduction/ (Fecha de acceso: 24.10.20159.

VAN DE WALLE, Bernie A.

2009 The heart of the Gospel : A.B. Simpson, the fourfold gospel, and late nineteenth-century evangelical theology. Eugene, Oregon: Pickwick Publications.

VAN SETERS, John

1983 In search of history : Historiography in the ancient world and origins of biblical history. New haven and London: Yale University Press.

WEBER, Max

1966 The sociology of religion. London: Methuen.

WIKIPEDIA

2014 Numinous. En: https://en.wikipedia.org/wiki/Numinous (Fecha de acceso: 24.10.2015). 